\title{
Low-frequency Topographic Amplification Effect of Lushan Earthquake Based on Three-dimensional Numerical Simulation
}

\author{
Feng Ligang ${ }^{1,2}$, Liu Qifang ${ }^{1,2}$ \\ ${ }^{1}$ Institute of Engineering Mechanics, China Earthquake Administration, Harbin, China \\ ${ }^{2}$ Key Laboratory of Earthquake Engineering and Engineering Vibration of China Earthquake Administration, Harbin, China
}

\section{Email address:}

hitfenglg@163.com (Feng Ligang), qifang_liu@126.com (Liu Qifang)

\section{To cite this article:}

Feng Ligang, Liu Qifang. Low-frequency Topographic Amplification Effect of Lushan Earthquake Based on Three-dimensional Numerical Simulation. Science Discovery. Vol. 7, No. 3, 2019, pp. 178-181. doi: 10.11648/j.sd.20190703.18

Received: April 22, 2019; Accepted: June 11, 2019; Published: June 15, 2019

\begin{abstract}
The study of the influence of terrain on ground motion is quantified as the amplification effect of terrain on ground motion. In this paper, we use the spectral element method to simulate the three-dimensional real terrain model, and select the aftershocks of the station ground motion observation records in the Lushan area as the source, and analyze the amplification effect of the three-dimensional topography on the ground motion of the Lushan earthquake in the low frequency range (1-5hz). In this paper, the Fourier model of the real terrain of Lushan and the half-space free model are used to calculate the Fourier amplitude spectrum. The results show that in the low frequency range, the ground fluctuations have a large variation, which will have complex effects on the propagation of seismic waves. Especially in the bulging mountain and depression terrain, the time course peak is large. In addition, this paper selects the mountain near the BXD station from the calculation area, and analyzes the terrain magnifying effect of the convex mountain. Under 2-3hz, the mountain slope near the source side has a magnification effect of 3-4 times.
\end{abstract}

Keywords: Three-dimensional Numerical Simulation, Spectral Element Method, Terrain Amplification Effect, Spectrum Ratio

\section{基于三维数值模拟的芦山地震低频地形放大效应}

\section{冯丽刚 ${ }^{1,2}$, 刘启方 ${ }^{1,2}$}

${ }^{1}$ 中国地震局工程力学研究所, 哈尔滨, 中国

${ }^{2}$ 中国地震局地震工程与工程振动重点实验室, 哈尔滨, 中国

邮箱

hitfenglg@163.com (冯丽刚)，qifang_liu $@ 126 . c o m($ 刘启方）

\begin{abstract}
摘要: 地形对地震动影响的研究, 量化表现为地形对地震动的放大效应方面。本文利用谱元法数值模拟做三维真实地 形模型, 选取芦山区域有台站地震动观测记录的余震做震源, 分析芦山地震在低频段 (1-5hz) 下的三维地形对地震动 的放大效应。本文对芦山真实地形三维模型与半空间自由模型的地震动模拟时程记录做傅氏振幅谱比分析,结果表明: 在低频段下, 地面起伏变化较大区域, 对地震波的传播会产生复杂效应, 尤其在凸起山体和凹陷地形区域, 时程峰值 较大。另外本文从计算区域中选取BXD台站附近山体, 具体分析凸型山体的地形放大效应, 在2-3hz下, 靠近震源一侧 山体坡面有3-4倍的放大效应。
\end{abstract}


关键词: 三维数值模拟, 谱元法, 地形放大效应, 频谱比

\section{1. 引言}

2013 年 4 月 20 日四川省芦山发生 $M_{w} 6.7$ 级地震, 这是 我国第一次在地表观测点获取到峰值超过 $1 \mathrm{~g}$ 的加速度记 录, 温瑞智等[1]都验证了局部地形影响是加速度记录较大 的主要原因。而在地震工程学中, 关于地形对地震动的影 响一直是研究的重点, 地震波在介质中的传播过程的精确 模拟也一直是大量研究者的突破点, 大量模拟方法也被应 用, 如有限拆分法、有限元法、伪谱法、谱元法等。通过 近几年的发展, 谱元法被Komatitsch[2-5]大量推广应用, 做出适用于二维与三维的地震波传播的模拟程序 SPECFEM2D和SPECFEM3D。我国研究者对于二维模型 以及二维场地放大效应做了大量的研究与分析, 得到了很 好的结果, 但是对于三维地形的地震动放大效应却很少。 实际上地震动的场地影响是在三维复杂地形情况下发生 的, 因此, 研究三维地形下对地震动的放大效应的研究是 很有必要的, 也是具有重要意义的。

本文利用SPECFEM3D法, 参考刘启方、于彦彦等 [6-11]的研究方法运行谱元法程序, 对芦山区域进行三维 真实地形数值模拟, 选取实际余震作为震源模拟分析低频 段下的地形放大。以下就三维地形放大效应展开详细研究。

\section{2. 数值模拟以及分析计算方法}

本文三维模型中地震动在介质中传播参考廖振鹏等 [12-13]波动方程来实现，介质弹性波传播的波动方程为:

$$
\begin{gathered}
\rho \ddot{u}=\nabla \cdot \sigma+\mathrm{f} \\
\sigma=\mathrm{c}: \nabla \mathrm{u}
\end{gathered}
$$

其中 $\mathrm{u}$ 是位移场, $\sigma$ 是二阶应力张量, $\mathrm{c}$ 是四阶弹性张 量, $\rho$ 是质量密度, $\mathrm{f}$ 是震源等外荷载。其中, 弹性张量 $\mathrm{c}$ 具有以下形式:

$$
\mathrm{c}_{\mathrm{ijkl}}=\lambda \delta_{\mathrm{ij}} \delta_{\mathrm{kl}}+\mu\left(\delta_{\mathrm{ik}} \delta_{\mathrm{jl}}+\delta_{\mathrm{il}} \delta_{\mathrm{jk}}\right)
$$

$\lambda$ 和 $\mu$ 分别为一阶与二阶拉梅常数。谱元法中的波动方 程为:

$$
\int_{\Omega} \rho w \cdot \ddot{u} d \Omega=\int_{\Gamma} w \cdot \sigma_{\Gamma} d \Gamma-\int_{\Omega} \nabla w \cdot \sigma d \Omega+\int_{\Omega} w f d \Omega
$$

式中， $\Omega$ 为计算区域的內域。对于自由地表, 满足应 力为零的边界条件, 因此自由地表內域节点的运动方程为:

$$
\int_{\Omega} \rho w \cdot \ddot{u} d \Omega=-\int_{\Omega} \nabla w \cdot \sigma d \Omega+\int_{\Omega} \mathrm{wf} d \Omega
$$

区域内部, 假设将计算域分解为 $n_{e}$ 个单元, $\Omega^{e}$ 为第 $\mathrm{e}$ 个单元, 每个单元上的波动方程为:

$$
\int_{\Omega_{e}} \rho w \cdot \ddot{u} d \Omega^{e}=-\int_{\Omega_{e}} \nabla w \cdot \sigma d \Omega^{e}+\int_{\Omega_{e}} \mathrm{wf} d \Omega^{e}
$$

本文的数值模拟是将计算区域分解为有限个单元, 每 个单元以数值积分配置节点, 在单元内传播地震动, 同时, 谱元法的单元波动方程并没有集成刚度矩阵, 以相互作用 力代之求解, 这样有利于单元的几何适应性, 可以更好地 模拟地震波的传播。从谱元法的波动方程中可以看出, 谱 元法自动满足自由地表应力为零的边界条件, 这样更加方 便对地表地形的模拟。

本文分析地形放大效应采用真实三维地形模拟的速 度记录的傅氏谱与半空间三维无地形模拟的速度记录的 傅氏 谱之比:

$$
\mathrm{R}_{\mathrm{i}}(\mathrm{w})=\frac{A_{i}(w)}{A_{0}(w)}
$$

其中 $A_{i}(w)$ 为真实三维地形模拟的速度记录的傅氏谱, $A_{0}(w)$ 为半空间三维无地形模拟的速度记录的傅氏谱。

\section{3. 三维模型与模拟分析}

\section{1. 三维数值模拟}

本文选取国家地形数据库中芦山真实地表地形作为 三维模型的地表面, 考虑到地表地形真实性与精确性, 地 表高程数据沿经度纬度方向隔 $50 \mathrm{~m}$ 取点, 地表网格考虑到 计算能力, 取 $300 \mathrm{~m} \times 300 \mathrm{~m}$ 网格大小, 为了方便计算, 垂直 方向分界面-5000m处按 $1: 3$ 做过度区划分网格进行三维建 模。图1所示为数值模拟的计算区域, 图 2 为三维模型, 包 括区域为东经 $102.7^{\circ}-103^{\circ}$, 北纬 $30.2^{\circ}-30.5^{\circ}$, 范围, 约 $28.6 \mathrm{~km} \times 33.2 \mathrm{~km}$, 垂直方向从地表到地下 $60 \mathrm{~km}$ 内的三维地 壳速度结构模型, 图中标记分别为所选取的余震 (星号标 记）以及三个台站 $51 \mathrm{BXD} 、 51 \mathrm{BXZ} 、 51 \mathrm{BXM}$ 的位置。与 此同时, 为了更好的分析芦山区域地形影响, 本文也对对 计算区域做半空间自由地表数值模拟, 与真实地形作对比。 模拟采用的地质速度构造综合吕坚等[14]地震测深与反演 的成果, 如表 1 所示数据。区域的 $\mathrm{Q}$ 值同一采用了 $\mathrm{Qp}=9000$ 和 $\mathrm{Q}=9000$ 。

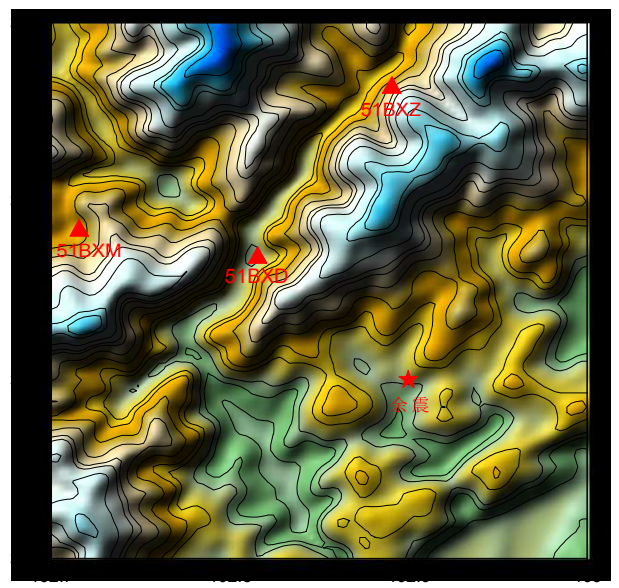

图1 数值模拟区域。 


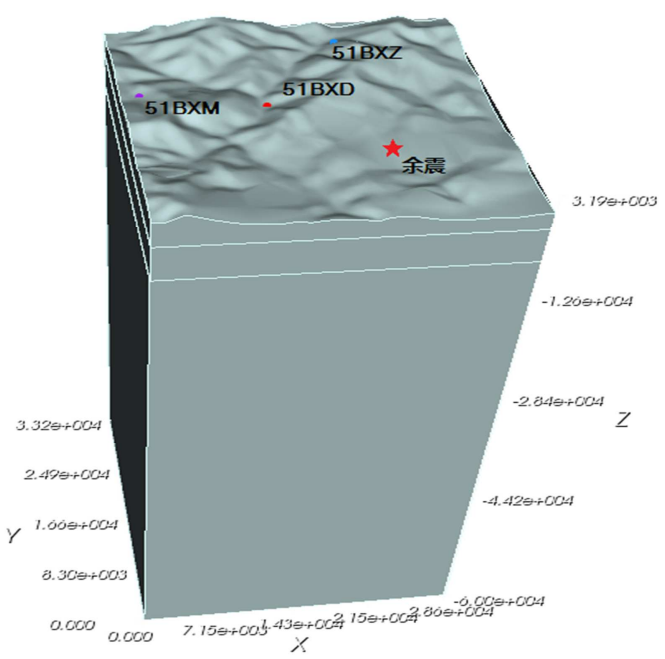

图2 芦山三维地形数值模型。

表1 计算区域地壳速度构造。

\begin{tabular}{llllll}
\hline 地壳分层 $/ \mathbf{k m}$ & 密度 $/\left(\mathbf{k g} / \mathbf{m}^{3}\right)$ & $\mathbf{V p} /(\mathbf{m} / \mathbf{s})$ & $\mathbf{V s} /(\mathbf{m} / \mathbf{s})$ & $\mathbf{Q p}$ & $\mathbf{Q s}$ \\
\hline 地表-2.94 & 2600 & 4880 & 2860 & 9000 & 9000 \\
$2.94-8.34$ & 2780 & 5800 & 3400 & 9000 & 9000 \\
$8.34-21.70$ & 2830 & 6040 & 3550 & 9000 & 9000 \\
$21.70-43.09$ & 3000 & 6820 & 3980 & 9000 & 9000 \\
$43.09-50$ & 3050 & 7000 & 4000 & 9000 & 9000 \\
$50-60$ & 3300 & 7850 & 4400 & 9000 & 9000 \\
\hline
\end{tabular}

本文选取了五条芦山余震作为震源模拟, 通过与国家 地震台网给出的五个余震在三个台站BXD、BXZ、BXM 处的速度观测记录作对比, 模拟结果近似一致。由于篇幅 限制这里取出其中一个余震在BXD台站的加速度记录, 余 震信息综合吕坚 $[14]$ 的地震反演参数设置为震源深度 $18 \mathrm{~km}$, 走向 $247^{\circ}$, 倾角 $57^{\circ}$, 滑动角 $121^{\circ}$ 。对余震记录进 行基线校正后, 做出加速度观测记录图, 如图3所示, 分 别是EW、NS、UD三方向的加速度记录, 其中EW方向加 速度峰值最高为 $52 \mathrm{~cm} / \mathrm{s}$, 远大于其他分量, 这与温瑞智等 [1]研究芦山主震在BXD台站处EW加速度峰值最大一致。 图4所示为观测记录与模拟记录做带通无相移滤波至 0.5-3hz的EW、NS方向速度对比图。通过对比, 在 $3 \mathrm{hz}$ 下, 三个台站处的观测记录与三维模拟记录速度曲线较为吻 合, 其中BXD台站模拟记录在地震波传播到达时刻 $(2 \mathrm{~s})$ 有一小段跳跃, 这可能是由于BXD台站复杂地形导致数值 模拟不是很完美, 但在波形的大致幅度上近似一致, 说 明数值模拟的三维模型较为符合真实情况地震波传播。

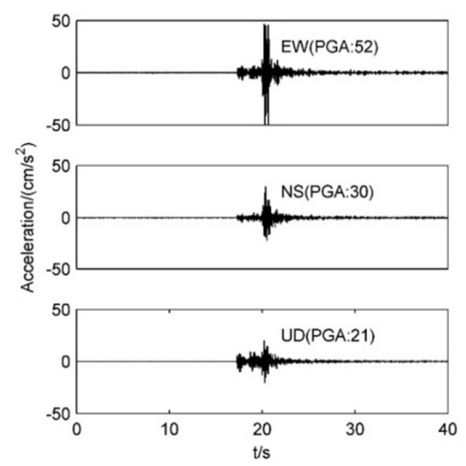

图3 震源在BXD台站处实际加速度记录。
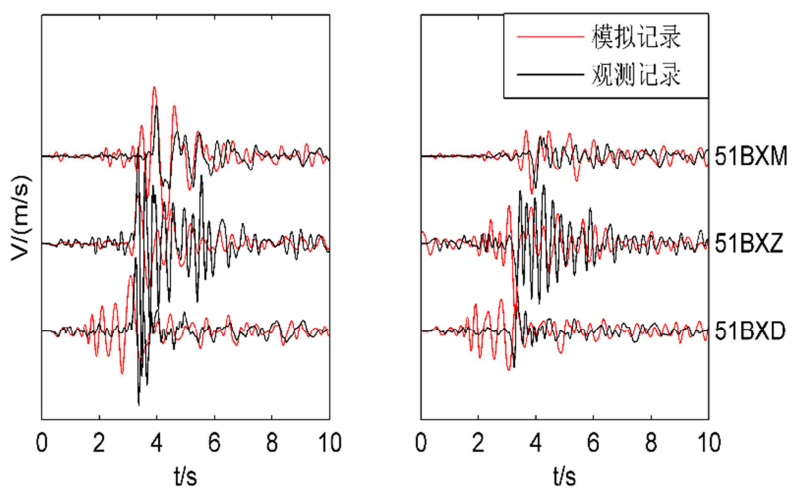

图4 模拟记录与观测记录在三个台站处EW(左)与NS（右）的速度图。

\section{2. 计算分析}

本文在模型范围内, 分别沿 $\mathrm{x}$ 和 $\mathrm{y}$ 方向隔 $300 \mathrm{~m}$ 在地表 上取观测点, 计算的速度记录带通滤波至 $0-3 \mathrm{~Hz}$, 图 5 为 计算区域在EW方向的速度记录峰值PGV分布。可以看出, 在地形复杂的区域（图5框选区域内）, 速度峰值变化明 显, 差异较大, 在一些凸起山体和凹陷地形区域, 峰值大 并且集中。相较来说, 在山体坡面上明显比山脚下的区域 峰值大, 这一现象表现明显, 这主要是因为山体对地震动 的放大影响。

为了具体研究低频段下的地形放大效应, 如图 5, 在 BXD台站附近取出一个台站到余震之间的山体作为研究 对象。从 $\mathrm{BXD}$ 台站到余震位置, 沿着图 5 中箭头依次在凸 形山体以BXD台站为起点取 23 个观测点, 图7所示 (从 BXD 台站到山下为止) 为所选点的山体大致形状。为了更好地 分析山体地形放大效应, 将观测点地形数值模拟与半空间 无地形模拟的速度记录沿着顺山向与垂直山体走向做坐 标转换, 图 5 中两箭头为新水平坐标。图6为真实三维地形 模拟的速度记录与半空间无地形三维模拟的速度记录在 新坐标下X方向（顺山方向）的傅式振幅谱之比。可以看 出, 谱比峰值大约在 $2.8 \mathrm{~Hz}$ 处, 从 $\mathrm{BXD}$ 台站处到 21 号观测 点, 谱比峰值是一个增大再减小的过程。在靠近震源一侧 的山体坡面, 从山脚到山顶, 谱比增大, 11 号与 16 号观测 点在 $2.8 \mathrm{~Hz}$ 的谱比峰值分别为为 3.5 和 4 , 地形放大效应明显, 而在山体背面谱比值较小, 如 $3 、 5 、 7$ 号观测点, 谱比峰 值在 2 以下，地形放大效应不明显。以上结果表明，在低 频段, 山体地形对地震动也有一定的放大效应。

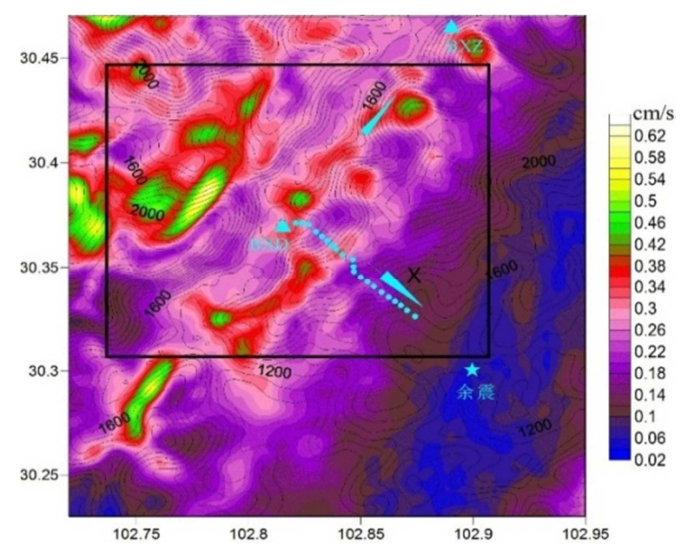

图5 PGV (EW方向)。 


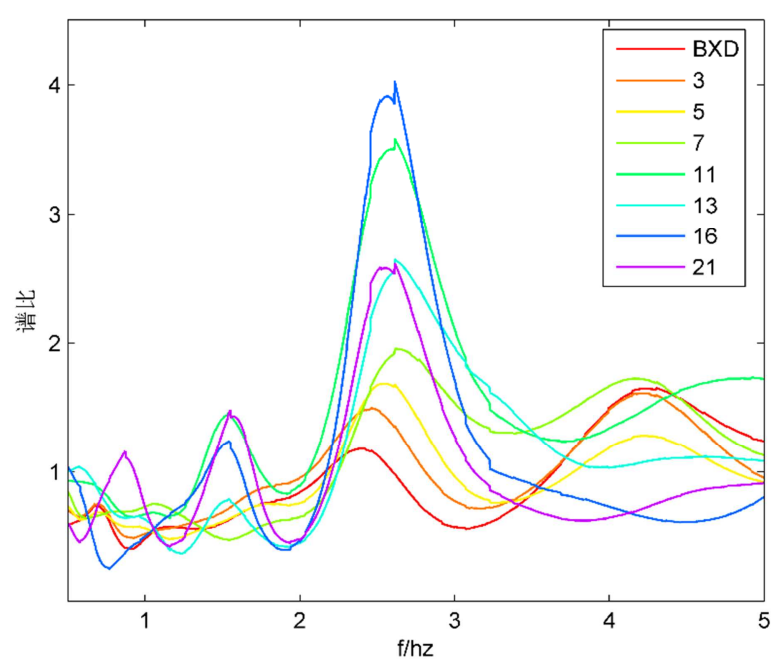

图6 地形模拟。

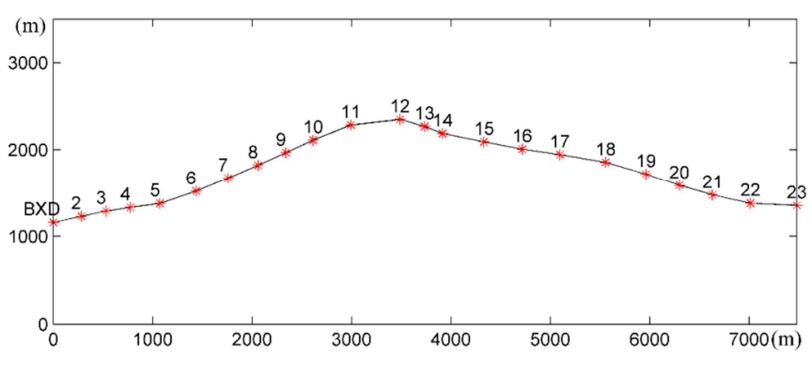

图7 山体走向。

\section{4. 结论}

本文采用三维真实地形数值模拟了芦山区域地震动 的传播影响, 并且对比半空间自由地形数值模拟, 利用谱 比法来分析低频段下地形对地震动的放大效应。结论表明: 在 $3 \mathrm{~Hz}$ 频段下, 地形对地震动仍有一定的影响, 复杂区域 的时程峰值变化剧烈, 表明地形起伏较大的地形对地震动 的影响也是较为复杂的, 其中凸起山体坡面和凹陷地形峰 值较大, 地形影响较为明显。具体分析山体对地震动的放 大效应, 结果表明, 山体正坡面（靠近震源一侧）地震动 放大效应明显，具体放大为 $200 \% \sim 400 \%$, 而在山体背坡面 对地面运动基本无放大。因此, 对于地震动影响方面, 本文建议也应考虑到低频段下地形对地震动影响。

\section{致谢}

本文为国家重大科技专项（2013zx06002001-09）的 阶段性成果之一。

\section{参考文献}

[1] 温瑞智, 任叶飞, 齐文浩, et al. 2013年4月20日芦山地震最 大加速度记录分析 [J]. 西南交通大学学报, 2013, 48(5)。

[2] Komatitsch D, Tromp J. Spectral-element simulations of global seismic wave propagationâ I. Validation[J]. Geophysical Journal International, 2002, 149(2):390-412.

[3] Komatitsch D, Tromp J . Spectral-Element Simulations of Global Seismic Wave Propagation-II. 3-D Models, Oceans, Rotation, and Self-Gravitation[J]. Geophysical Journal of the Royal Astronomical Society, 2002, 150(1):303-318.

[4] Lee S J, Chen H W, Liu Q, et al. Three-Dimensional Simulations of Seismic-Wave Propagation in the Taipei Basin with Realistic Topography Based upon the Spectral-Element Method [J]. Bulletin of the Seismological Society of America, 2008, 98(1):253-264.

[5] Assimaki, D. Effects of Local Soil Conditions on the Topographic Aggravation of Seismic Motion: Parametric Investigation and Recorded Field Evidence from the 1999 Athens Earthquake [J]. Bulletin of the Seismological Society of America, 2005, 95(3):1059-1089.

[6] 刘启方, 于彦彦, 章旭斌. 施甸盆地三维地震动研究 [J]. 地 震工程与工程振动, 2013, 33(04):054-60。

[7] 刘启方, 李雪强, 孙平善. 施甸盆地三维速度结构模型研 究 [J]. 地震工程与工程振动, 2013, 33(03):088-94。

[8] 刘启方, 李雪强. 唐山大地震近场宽频带地震动模拟 [J]. 地震工程与工程振动, 2011, 31(5)。

[9] 张建毅, 薄景山, 王振宇, et al. 汶川地震局部地形对地震 动的影响 [J]. 自然灾害学报, 2012, 21(03):164-169。

[10] 于彦彦. 三维沉积盆地地震效应研究 [J]. 国际地震动态, 2017(6):33-35。

[11] 蒋涵. 三维地形特征与地震动放大系数的相关性研究 [D]. 中国地震局地球物理研究所, 2014。

[12] 廖振鹏. 工程波动理论导论[M]. 科学出版社, 2002。

[13] 廖振鹏，杨柏坡，袁一凡. 三维地形对地震地面运动的影 响 [J]. 地震工程与工程振动, 1981(1):59-80。

[14] 吕坚, 王晓山, 苏金蓉, et al. 芦山7.0级地震序列的震源位 置与震源机制解特征 [J]. 地球物理学报, 2013, 56(5):1753-1763。 